\title{
Estimation of coefficient of lateral stress used in the calculation of loads on buried structures
}

\author{
Talia S. da Silva1, Mohammed Z.E.B. Elshafie, PhD $^{2}$, and \\ Gopal S.P. Madabhushi, $\mathbf{P h D}^{3}$
}

\begin{abstract}
${ }^{1}$ Research student, Schofield Centre, Department of Engineering, University of Cambridge, Trumpington Street, Cambridge, United Kingdom, CB2 1PZ; e-mail: tsd30@cam.ac.uk

${ }^{2}$ Lecturer, Department of Engineering, University of Cambridge, Trumpington Street, Cambridge, United Kingdom, CB2 1PZ; e-mail: me254@cam.ac.uk

${ }^{3}$ Professor \& Head of Geotechnical and Environmental Research Group, Schofield Centre, Department of Engineering, University of Cambridge, Trumpington Street, Cambridge, United Kingdom, CB2 1PZ; e-mail: mpsg1@cam.ac.uk
\end{abstract}

\begin{abstract}
The average vertical pressure on a buried structure can be calculated using the silo theory, which assumes the translation of a vertical prism of soil above the structure that is resisted by friction on the sides of the prism. One of the key assumptions made is the value of the coefficient of lateral stress, $K$. In this study, an assumption regarding the rotation of principal stresses in the yielding soil has been used to calculate the average coefficient of lateral stress acting at the side of a prism of yielding soil above the horizontal buried structure. The calculated value using the proposed method agrees well with experimental observation made in literature for the value of $K$, and is suggested for use in the estimation of loads on buried structures, where it is expected that the structure will yield relative to a stiff body of soil.
\end{abstract}

\section{INTRODUCTION}

Many studies have been conducted to estimate the vertical pressure acting on a trapdoor in active arching. These are developed from the silo theory of Janssen (1895), which considers friction on a vertical wall that reduces the vertical pressure at the base of the silo. In trapdoor studies, the failure mechanism above the trapdoor is approximated to a prism with vertical sides in the ultimate failure state. The most prominent early work was conducted by Terzaghi (1946), who investigated arching in ideal soils in trapdoors, which can be used as an approximation for a buried structure in a stiff soil.

The derivation for the pressure acting on the base considers an infinitesimal layer of soil subject to its own weight, with vertical sliding surfaces supported by frictional forces. A schematic representation of the plane-strain problem and the resulting free body diagram used to solve for the vertical stress on the yielding structure is shown in Figure 1. 
The normal stress acting on the sliding plane, $\sigma_{a h}$, is equal to $K \sigma_{v}$, where $\sigma_{v}$ is the vertical stress in the soil and $K$ is the coefficient of lateral stress. The Mohr-Coulomb model is used for a cohesionless soil, and the shear force on the slip plane is given by: $\tau_{w}=\tan \delta$, where $\delta$ is the mobilized angle of friction on the plane. $\delta \leq \phi$, where $\phi$ is the internal angle of friction of the soil.

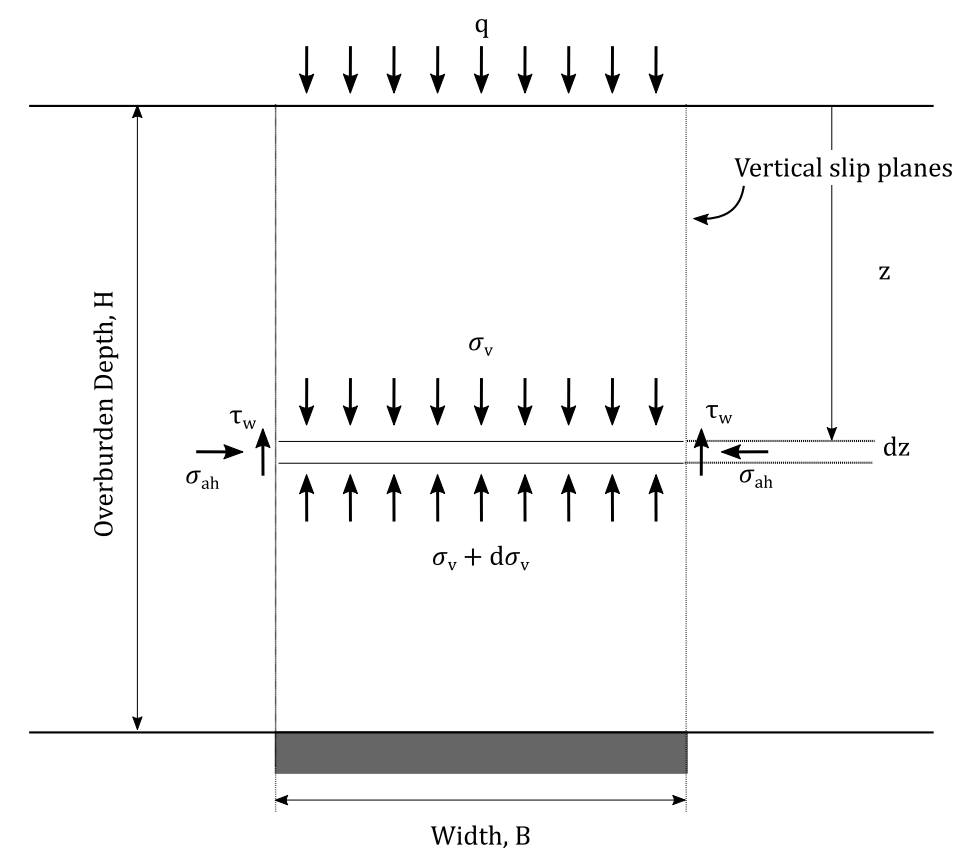

Figure 1. Arching above a yielding trapdoor (after Terzaghi, 1946)

Vertical equilibrium of a flat horizontal element of differential height gives:

$\frac{d \sigma_{v}}{d z}=\gamma-K \sigma_{v} \frac{2 \tan \delta}{B}$

The integration of Equation 1 from the surface with a pressure of $q$ to the base at a depth of $H$ gives the vertical pressure on the trapdoor as:

$\sigma_{v, \text { base }}=\frac{\gamma B}{2 K \tan \delta}\left(1-e^{-2 K \tan \delta H / B}\right)+q e^{-2 K \tan \delta H / B}$

In order to apply this equation, an assumption of the coefficient of lateral stress, $K$, acting on the failure surface needs to be made. Terzaghi indicated that the assumption of a value of unity leads to good agreement with experimental results. Some applications have suggested the use of the active coefficient of lateral stress, $K_{a}$ : for example, Marston and Anderson, (1913)). As described by Handy (1985), these sliding planes have frictional forces on them to support the failure equilibrium (shear forces), and thus $K \neq K_{a}$ as the latter implies the existence of principal stresses on the boundary. 
To overcome this limitation, Handy (1985) considered the rotation of principal stresses at the sliding planes. If the rotation of principal stresses across a flat differential element is known, the average vertical stress on the element can be determined, and therefore the average coefficient of lateral stress that is acting at the boundary, $K_{\text {ave }}$, in order to maintain equilibrium of the element. In the derivation by Handy (1985), this rotation assumed a concave trajectory of minor principal stresses in the shape of a circular arc. This method was developed further by Paik and Salgado (2003) in the estimation of active earth pressure behind retaining walls with a consideration of the arching effect, with an improvement in the calculation of $K_{\text {ave }}$.

The analytical solution presented in this paper has been adapted for the evaluation of the active translation of a trapdoor below a cohesionless soil, with the critical change of the assumption of a convex trajectory of the major principal stresses, which is deemed more appropriate than the original suggestions made by Handy (1985) and Paik and Salgado (2003).

\section{ANALYTICAL SOLUTION}

\section{Rotation of major prinicipal stresses}

Because the vertical equilibrium of the differential element in Figure 1 is achieved by friction acting on the slip plane, there is a shear force acting along this plane and the principal stresses must therefore be inclined at an angle relative to this plane. Due to the symmetry of the problem, there are no shear stresses on the centreline of the trapdoor.

When there is an active translation of a trapdoor below a granular material, the material forms an 'arch' over the yielding area to support the load (Terzaghi, 1943). This arch can be considered to follow the trajectory of the major principal stresses, as this is the path where the loads will be transferred. This can be verified from experimental and numerical observations of the phenomena; see for example the discrete element modelling and plotting of principal strain directions in Chevalier et al. (2012).

This requires that on the centreline, the major principal strain is horizontal and the minor principal strain is vertical; the soil in this region is in a passive state of failure.

This is in contrast to the assumptions that have been made in the analysis by Handy (1985) and followed by Paik and Salgado (2003) which suggest that the major principal stress is vertical at the centreline. The assumption of a passive state of failure leads to the requirement that the trajectory of the major principal stresses will make a convex arch from an inclined angle $\theta$ to the horizontal at the sliding plane, to horizontal along the centreline (no inclination). The minor principal stresses are applied normal to this convex arch. A representation of this problem definition is shown in Figure 2 and shown in more detail in Figure 3. 


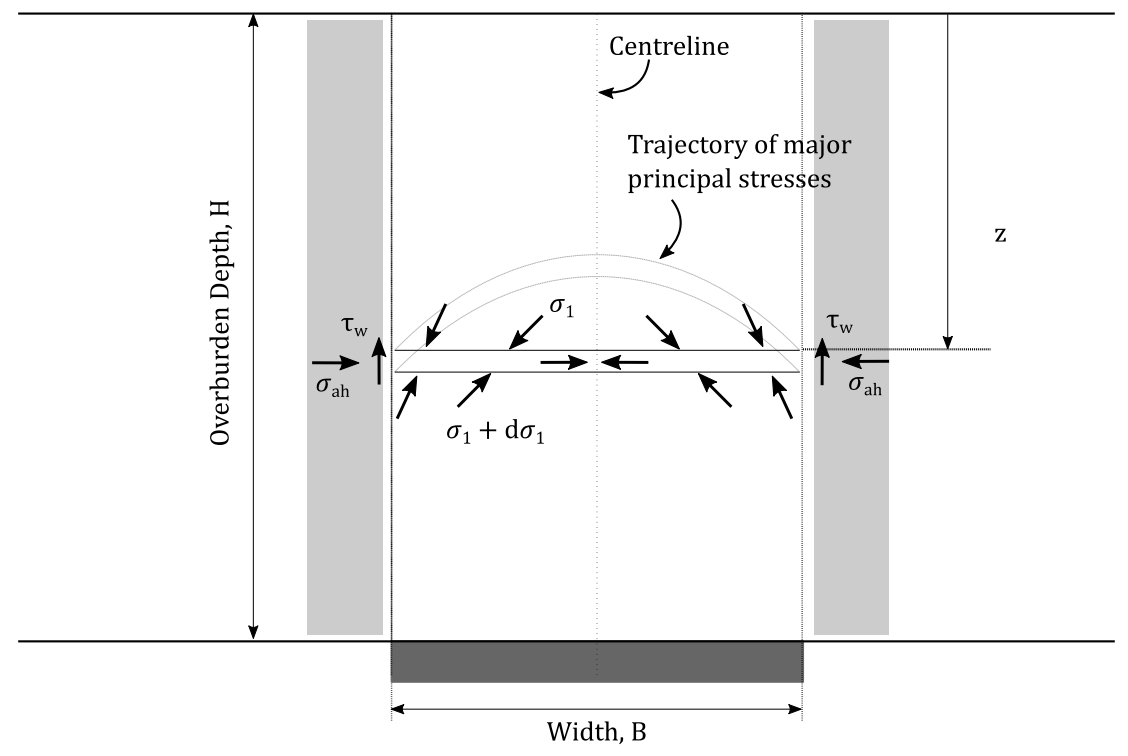

Figure 2. Trajectory of major principal stresses in yielding soil
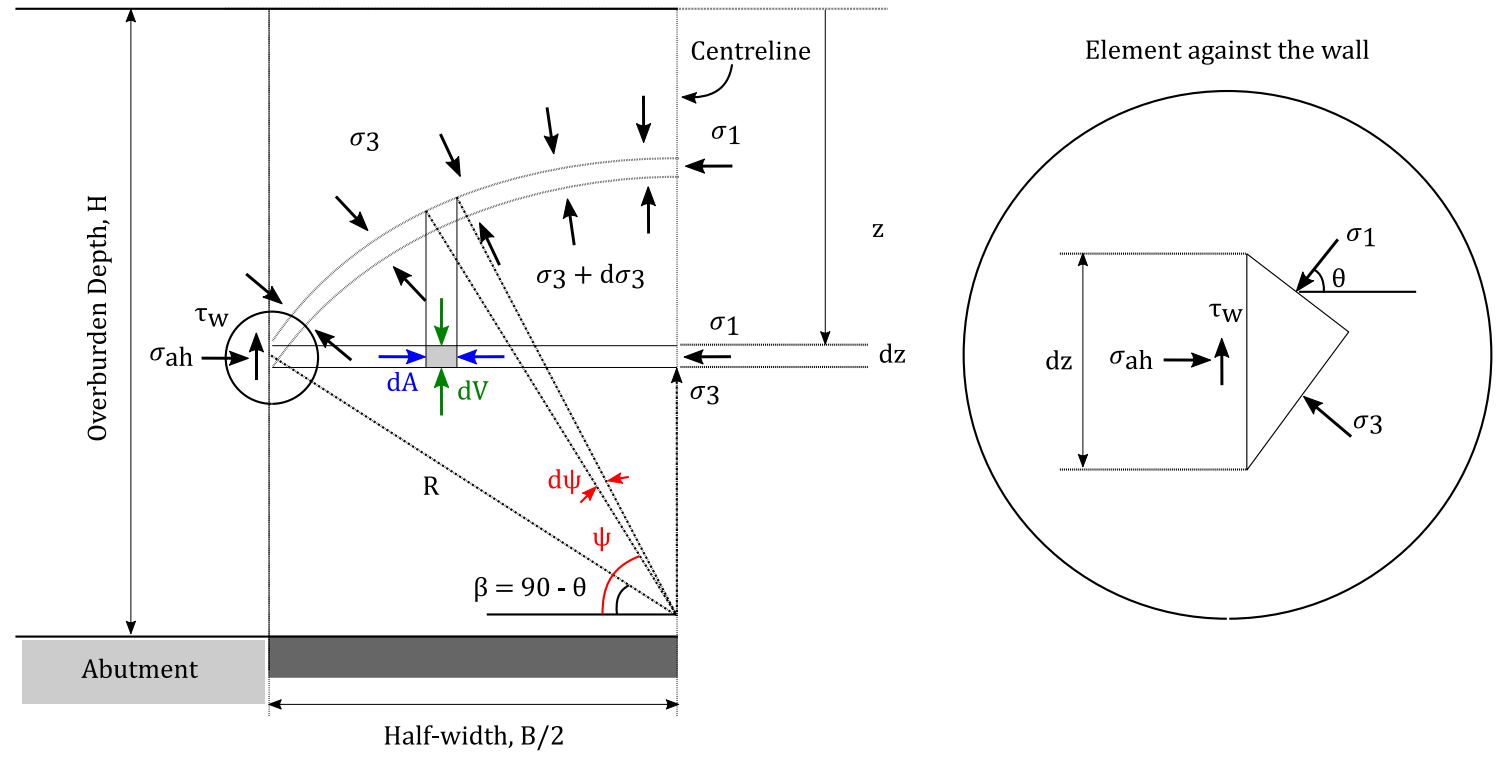

Figure 3. Differential flat element with rotation of major principal stresses

The angle of rotation of the major principal stress at the sliding plane can be calculated from the soil properties. Equilibrium of the triangular element at the edge of the wall, shown in the inset in Figure 3, gives:

$\Sigma F_{x}=0 \quad \therefore \sigma_{a h} d z=\left(\sigma_{1} d z \cos \theta\right) \cos \theta+\left(\sigma_{3} d z \sin \theta\right) \sin \theta$ 
$\sigma_{a h}=\sigma_{1} \cos ^{2} \theta+\sigma_{3} \sin ^{2} \theta$

If this is written in terms of the minor principal stress rotation from the horizontal, $\beta\left(\beta=90^{\circ}-\theta\right)$, then:

$\sigma_{a h}=\sigma_{1} \sin ^{2} \beta+\sigma_{3} \cos ^{2} \beta$

At any point along the width of the differential element shown in Figure 3, the minor principal stress rotation is $\psi$. This is the angle between the tangent to the trajectory and the vertical, and the horizontal stress at this point can be written as.

$\sigma_{h}=\sigma_{1} \sin ^{2} \psi+\sigma_{3} \cos ^{2} \psi$

Dividing this equation by $\sigma_{1}$, gives:

$\frac{\sigma_{h}}{\sigma_{1}}=\sin ^{2} \psi+\frac{1}{N} \cos ^{2} \psi$

where $N$ is the ratio of major to minor principal stresses, which is equal to the passive coefficient of lateral stress, $N=1+\tan ^{2}(45+\phi / 2)$.

From the Mohr's circle of stresses: $\sigma_{1}+\sigma_{3}=\sigma_{\mathrm{v}}+\sigma_{\mathrm{h}}$. Dividing this by $\sigma_{1}$, and solving through, the ratio of the vertical stress to the principal stress at this same point can be found as:

$\frac{\sigma_{v}}{\sigma_{1}}=\cos ^{2} \psi+\frac{1}{N} \sin ^{2} \psi$

The horizontal and vertical stress at any point along the width of the differential element can be calculated using Equations 6 and 7 if the angle $\psi$ is known or assumed across the width of the element.

\section{Angle of rotation of major principal stress at the sliding plane, $\theta$}

The Mohr circle of stress for the yielding soil with an internal angle of friction, $\phi$ and no cohesion is shown in Figure 4, assuming that the soil is in a limiting state of failure (i.e. the circle is tangential to the Mohr-Coulomb failure line). The mobilized friction on this slip plane, $\delta$, is shown in the diagram. Using the sign convention for shear stresses in soil mechanics, counterclockwise shear stresses acting on an element are positive. From the free body diagram in Figure 3, we know that the soil above the trapdoor is moving downwards, and the shear stress is acting upwards on 
the plane to support the soil. The shear stresses on this plane are clockwise and therefore considered negative.

For an angle of mobilized friction less than the maximum internal angle of friction, the Mohr circle shows two possible solutions for the normal and shear stresses acting on the wall. For a vertical wall, the intersection at the lower normal stress tends towards an active state in the soil and the intersection at the higher normal stress tends towards to a passive state of failure in the soil with $\sigma_{h}>\sigma_{v}$. The Mohr circle in Figure 4 shows the 'passive' intersection point.

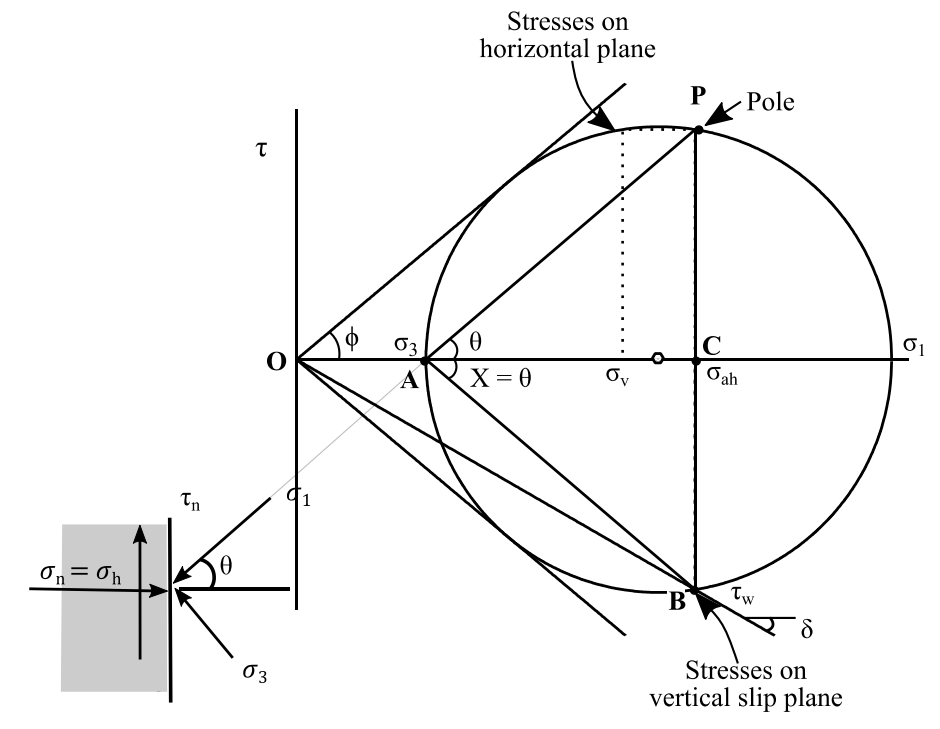

Figure 4. Mohr circle of soil in differential flat element

From triangles $\mathrm{OBC}$ and $\mathrm{ABC}$ in the Mohr circle:

$\tau_{w}=\sigma_{a h} \tan \delta=\left(\sigma_{a h}-\sigma_{3}\right) \tan \theta$

$\tan \theta=\frac{\sigma_{a h}}{\sigma_{3}}\left(\frac{\sigma_{a h}}{\sigma_{3}}-1\right) \tan \delta$

Substituting $\sigma_{a h} / \sigma_{3}$ from Equation 3 into Equation 8 leads to the following equation, from which the solution of $\theta$ can be obtained (Equation 10).

$\tan \theta=\frac{N+\tan ^{2} \theta}{N-1} \tan \delta$

$\theta=\tan ^{-1} \frac{(N-1) \pm \sqrt{(N-1)^{2}-4 N \tan ^{2} \delta}}{2 \tan \delta}$

As there are two potential intersection points for the mobilized friction, there are two solutions for $\theta$. The assumption made in the rotation of principal stresses is that the soil is in a passive state of failure on the centreline. It is therefore expected that at the failure plane, the soil will tend towards 
the passive state of failure, and the smaller solution for $\theta$ from Equation 10 should be used. If $\delta=\phi$, then there is only one solution, and $\theta=45^{\circ}+\phi / 2$.

\section{Average coefficient of lateral stress, $K_{\text {ave }}$}

The average lateral stress ratio, $K_{\text {ave }}$, acting on the vertical slip surface can be calculated by dividing the horizontal stress at the wall by the average vertical stress acting across the differential flat element.

Once the angle of rotation of the major principal stress at the boundary is known, an assumption can be made about the distribution of this rotation across the flat differential element in Figure 3. Assuming that the trajectory of the major principal stresses takes the shape of a circular arc, the vertical force, $d V$, acting on an element of differential width $d A$ in this element is:

$d V=\sigma_{v} d A=\sigma_{1}\left(\cos ^{2} \psi+\frac{1}{N} \sin ^{2} \psi\right)(R d \psi \sin \psi)$

The total width of the element is $B / 2$, and $B / 2=R \cos \beta$. The half width is used as the integration is made from the slip plane to the centerline where the rotation of the minor principal stresses are known. The average vertical stress, $\overline{\sigma_{v}}$, is the sum of the total vertical force divided by the width. The vertical force is integrated from $\psi=\beta$ at the vertical sliding plane, to $\psi=\pi / 2$ at the centerline where the principal stresses act on vertical and horizontal planes.

$\overline{\sigma_{v}}=\frac{V}{B / 2}=\frac{1}{B / 2} \int d V=\int_{\beta}^{\frac{\pi}{2}} \sigma_{1}\left(\cos ^{2} \psi+\frac{1}{N} \sin ^{2} \psi\right) \frac{\sin \psi}{\cos \beta} d \psi$

Integration of this equation gives the result:

$\overline{\sigma_{v}}=\sigma_{1} \frac{(N-1) \cos ^{2} \beta+3}{3 N}=\sigma_{1} \frac{(N-1) \sin ^{2} \theta+3}{3 N}$

Dividing Equation 3 by this equation above yields the result for the average coefficient of lateral stress on the vertical sliding plane considering the rotation of principal stresses occurring in the soil which is in a limit equilibrium failure state.

$K_{\text {ave }}=\frac{\sigma_{a h}}{\overline{\sigma_{v}}}=\frac{\sigma_{1} \cos ^{2} \theta+\sigma_{3} \sin ^{2} \theta}{\sigma_{1}\left((N-1) \sin ^{2} \theta+3\right)} 3 N=\frac{3\left(N \cos ^{2} \theta+\sin ^{2} \theta\right)}{3+(N-1) \sin ^{2} \theta}$

This average value for the coefficient of lateral stress can be substituted into Equation 2 to calculate the vertical stress at the base of the prism of soil, i.e. on the buried structure. 


\section{COMPARISON TO EXPERIMENTAL RESULTS}

\section{K-values from active arching experiments literature}

Several experimental investigations into the behavior of soil above a trapdoor have been conducted and are available in literature. Many of these have either measured the $K$-value in the arching process directly, or estimated it from back calculations from measured vertical pressure at the trapdoor.

In his trapdoor tests, Terzaghi (1936) measured a $K$-value of 1.0 immediately above the centerline, to a value of 1.6 one a half times the width of the trapdoor above the centerline; the soil internal angle of friction is approximately $30^{\circ}$. As highlighted by Evans (1983), based on these

results, Terzaghi (1946) describes $K$ as an empirical constant and recommends that it be taken to equal 1.0 for analysis purposes.

Further investigation was conducted by Evans (1983), who also measured the K-value at the trapdoor in physical model tests. These results showed that $K$ was not constant through the test: a peak value of 1.2 was obtained at the point when the minimum load on the trapdoor was measured, which reduced to a final constant value of approximately 0.8 as the translation of the trapdoor continued. The angle of internal friction of the soil used is given as $33^{\circ}$.

Chevalier et al. (2012) also made the observation that a value greater than unity was more appropriate than previous suggestions of using the active lateral stress ratio or values of unity. An appropriate value of $K$ was determined from fitting the analytical solution to experimental pressure measurements with arching in sands and gravels.

$$
\begin{aligned}
& \text { Sand: } K=1.17 ; \phi_{\text {peak }}=49^{\circ} ; \phi_{\text {critical }}=39^{\circ} \text {. } \\
& \text { Gravel: } K=1.40 ; \phi_{\text {peak }}=54^{\circ} ; \phi_{\text {critical }}=40^{\circ} \text {. }
\end{aligned}
$$

\section{Predictions from analytical solution}

The predicted $K$-value to be used in the analysis of the vertical stress acting on a buried structure can be calculated from the soil properties using Equations 10 and 14 assuming that the soil fails with vertical slip planes, and that a circular arch is formed. If the mobilized friction on the sliding plane is equal to the angle of internal friction, the resulting average value of the coefficient of lateral stress on the sliding plane is plotted in Figure 5.

If $\delta<\phi$, then the angle of rotation of the major principal stress will be less than if $\delta=\phi$ (see Mohr circle in Figure 4). If the pole of the circle is to the right of the circle center, then intuitively, it can be seen that this results in a K-value that will be greater than that shown in Figure 
5 as the rotation of the major principal stress will be flatter and approaching that of the fully passive state.

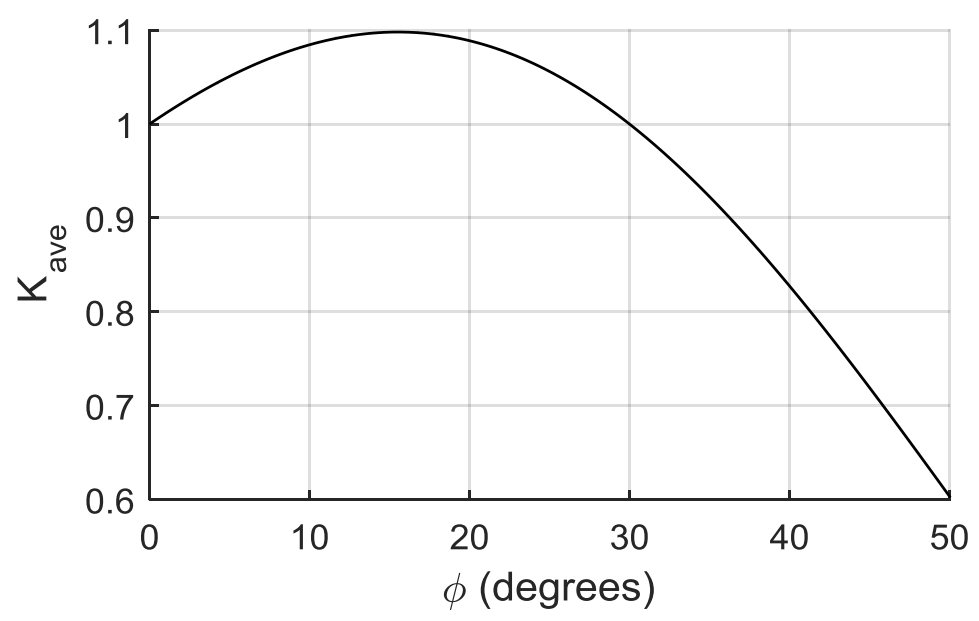

Figure 5. $K_{\text {ave }}$ as a function of $\phi$, assuming $\delta=\phi$

The $K_{\text {ave }}$ values for the experimental results from literature have been calculated using the above method, and the results are presented in Table 1 . Where only a single internal angle of friction is reported, it has been assumed that $\delta=\phi$. For the results from Chevalier et al. where the peak and critical state angles of friction have been reported, it has been assumed that $\phi=\phi_{\text {peak }}$ and $\delta=\phi_{\text {critical }}$.

Table 1. Mohr circle of soil in differential flat element

\begin{tabular}{|l|c|c|c|c|c|}
\hline Reference & $\phi\left(^{\circ}\right)$ & $\delta\left(^{\circ}\right)$ & $\theta\left(^{\circ}\right)$ & $K$ from literature & Calculated $K_{\text {ave }}$ \\
\hline Terzaghi (1943) & 30 & 30 & 60 & 1.00 & 1.00 \\
\hline Evans (1983) & 33 & 33 & 61.5 & $1.2(0.8)$ & 0.96 \\
\hline $\begin{array}{l}\text { Chevalier et al. } \\
(2012) \text {, sand }\end{array}$ & 49 & 39 & 55.08 & 1.17 & 1.27 \\
\hline $\begin{array}{l}\text { Chevalier et al. } \\
(2012) \text {, gravel }\end{array}$ & 54 & 40 & 52.01 & 1.40 & 1.53 \\
\hline
\end{tabular}

The calculated $K_{\text {ave }}$ for Terzaghi matches exactly with his recommendation for the use of a value of unity in the analysis of vertical stresses on the trapdoor; these equations used give a numerical basis based on soil mechanics principles and an understanding of the rotation of principal stresses to get to this previously empirically estimated factor. The calculated value for the case presented by Evans (1983) is the average of the observed values in the test. If the angle of friction given is the critical angle of friction and the peak angle is higher than $33^{\circ}$, then values for $K_{\text {ave }}$ close to the observed values in the tests can be calculated. 
The match between the back calculated values by Chevalier et al. (2012) and the calculated value using the equations in this paper is excellent, and suggests that the theory presented can be used in analysis with a good prediction of the expected vertical stresses on buried structures.

\section{CONCLUSION}

One of the key assumptions made in the estimation of loads on buried structures when the silo theory of Janssen (1895) is applied is the assumption of an appropriate value for the coefficient of lateral stress, $K$. Previous analysis has suggested the use of an empirical factor (Terzaghi, 1943), the active coefficient of lateral stress (Marston and Anderson, 1913), or an average based on the trajectory of minor principal stresses (Handy, 1985), amongst others.

Based on the idea of an arch supporting the yielding soil above a buried structure, this investigation has considered the assumption of the rotation of principal stresses with a trajectory of major principal stresses. The formation of vertical slip surfaces has been assumed as per the silo theory. The principal stress is passive above the centerline, and tending towards passive at the vertical slip surface.

A series of equations have been presented which allow the calculation of the rotation of the major principal stress at the slip surface, as well as the average coefficient of lateral stress at this boundary assuming a circular rotation of major principal stresses. The equations were used to compare to the results of estimates or measurements of $\mathrm{K}$ that have been presented by other researchers in the literature. An excellent match was observed, and this method of the estimation of the coefficient of lateral stress is therefore expected to give a more accurate prediction of the loads acting on buried structure when the structure is included in a stiff surrounding soil.

\section{ACKNOWLEDGEMENTS}

The first author would like to acknowledge the Gates Cambridge Trust for funding to conduct this research.

\section{REFERENCES}

Chevalier, B., Combe, G., \& Villard, P. (2012). Experimental and discrete element modeling studies of the trapdoor problem: influence of the macro-mechanical frictional parameters. Acta Geotechnica, 7(1), 15-39.

Evans, C.H. (1983). An examination of arching in granular soils. M.Sc. Thesis. Massachusetts Institute of Technology, Cambridge, USA.

Handy, R.L. (1985). The arch in soil arching. Journal of Geotechnical Engineering, 111(3), pp.302-318. 
Janssen, H. A. (1895). "Versuche über Getreidedruck in Silozellen." Z. d. Vereins deutscher Ingenieure, 39, 1045 (in German; partial English translation in Proc. Inst. Civil Eng. Lond., 1896, 553).

Marston, A., \& Anderson, A. O. (1913). The Theory of Loads on Pipes in Ditches: And Tests of Cement and Clay Drain Tile and Sewer Pipe. Iowa State College of Agriculture and Mechanic Arts.

Paik, K.H. and Salgado, R., (2003). Estimation of active earth pressure against rigid retaining walls considering arching effects. Geotechnique, 53(7), pp.643-654.

Terzaghi, K. (1936). Stress distribution in dry and saturated sand above a yielding trapdoor. Proc., First Int. Conf., Soil Mechanics and Foundation Engineering, Graduate School of Engineering, Harvard University, Cambridge, MA, 307-311.

Terzaghi, K. (1943). Theoretical soil mechanics. New York, Wiley. 